\title{
Evaluación de conocimientos y autonomía para la transición de adolescentes hacia la atención de adultos
}

\author{
Assessment of knowledge and autonomy for the transition from adolescent toward \\ adult care
}

\author{
Francisco Funes D. ${ }^{a}$, Francisca León L. ${ }^{b}$, Romina Valenzuela C. ${ }^{c}$
}

aPediatra Adolescentólogo. Unidad de Adolescencia y Transición, Hospital Luis Calvo Mackenna. Santiago, Chile

bPsicóloga. Unidad de Psicología, Hospital Luis Calvo Mackenna. Santiago, Chile.

'Enfermera Universitaria. Unidad de Investigación, Departamento de Pediatría y Cirugía Infantil Área Oriente, Universidad de Chile. Santiago, Chile

Recibido: 21 de noviembre de 2019; Aceptado: 6 de julio de 2020

¿Qué se sabe del tema que trata este estudio?

El paso de la atención de adolescentes con patología crónica desde pediatría a la atención de adultos requiere de un proceso de transición. Es recomendable contar con instrumentos que ayuden a evaluar el grado de preparación de estos pacientes.

\section{¿Qué aporta este estudio a lo ya conocido?}

Se entrega un instrumento clínico con contenido validado por expertos que permite evaluar el grado de preparación de adolescentes con patología crónica para su traslado a la atención de adultos.

\section{Resumen}

Cada vez más niños con enfermedades complejas logran sobrevivir requiriendo el paso de una atención pediátrica a una de adultos. Es fundamental contar con herramientas que permitan conocer el grado de preparación del paciente para este traslado. Objetivo: Crear un cuestionario local para medir el estado de preparación para la transición de adolescentes con enfermedad crónica y someterlo a pruebas de validación. Pacientes y Método: Basado en cuestionarios internacionales se diseñó un instrumento de auto-reporte que se sometió a validez de contenido por expertos, y luego a pruebas de comprensión y factibilidad en grupo piloto. Posteriormente se realizó validación de constructo y fiabilidad utilizando análisis factorial tras ser aplicado en adolescentes con enfermedad crónica. Resultados: Tras el análisis por 11 expertos y piloto de 8 pacientes se obtuvo un instrumento que fue respondido en forma completa por 168 adolescentes. Edad promedio 14,4 años. Tras la validación de constructo se genera un instrumento de 24 ítems de alta relevancia clínica, con 9 ítems con resultados psicométricos aceptables, los que se destacan en el cuestionario final. Conclusión: Se presenta un cuestionario de auto-reporte para medir el estado de preparación de los adolescentes para la transición a servicios de adultos. Las propiedades psicométricas del instrumento resultaron insuficientes para su validacion, ya que sólo se comprueba la validez de constructo y confiabilidad para 9 de los 24 ítems.
Palabras clave: Transición a Servicios de Adultos;

Encuestas y Cuestionarios; Auto-reporte; Enfermedad Crónica; Adolescente 


\begin{abstract}
In the last decades more and more children survive with complex health conditions, requiring a transition from pediatric to adult care. It is essential to have instruments that provide information on the level of preparation of patients for this process. Objective: To create and validate a questionnaire to measure the readiness status of adolescent patients with chronic diseases in the transition process. Patients and Method: Based on international questionnaires, a self-report instrument was designed which was subjected to content validity by experts, and then to comprehension and feasibility tests in a pilot group. Subsequently, construct and reliability validation were performed through a factorial analysis after applied it to adolescents living with a chronic illness. Results: After the analysis made by 11 experts and the pilot group with 8 patients, we obtained an instrument that was fully answered by 168 teenagers (Average age 14.4 years). After construct validation, a 24 -items instrument of high clinical relevance was developed, with 9 items with acceptable psychometric properties, which were highlighted in the final questionnaire. Conclusion: a self-report instrument aimed to measure the readiness of adolescents during the transition process to adult care is presented. The reported psychometric properties of the instrument were insufficient to consider it validated since the construct validity and reliability were only checked for 9 of the 24 items.
\end{abstract}

Keywords:

Transition to Adult

Care;

Surveys and

Questionnaires;

Self-report;

Chronic Illness;

Adolescent

\section{Introducción}

Cada vez más niños con enfermedades complejas logran sobrevivir llegando a la adolescencia y a la adultez, requiriendo el paso de una atención pediátrica a una de adultos ${ }^{1}$. Este hito crea inquietud en los pacientes, familia y pediatras ${ }^{2,3}$, y se ha visto que puede tener un impacto negativo en la salud de los pacientes ${ }^{4-7}$. A esto se suma que en Chile esta transferencia ocurre en plena adolescencia, lo que tampoco es recomendado ${ }^{8}$.

Sociedades científicas, entre ellas la Rama de Adolescencia de la Sociedad Chilena de Pediatría, han manifestado que la transferencia del cuidado hacia los servicios de adultos debe realizarse mediante servicios de transición que realicen un proceso dinámico y permanente, enfocado a satisfacer las necesidades individuales del paciente en el paso de la niñez a la vida adulta con el fin de maximizar su potencial y funcionamiento a través de la vida. Este proceso debe ser suministrado mediante servicios de alta calidad, apropiados para el desarrollo, sin interrupciones y centrado en el paciente ${ }^{8-12}$.

Dada su complejidad, el proceso de transición requiere que los adolescentes adquieran conocimientos y habilidades que resulten en un mejor autocuidado de sus enfermedades, lo que se recomienda ir fomentando y evaluando en los controles de salud. Si bien la práctica clínica individual no exige necesariamente el uso de cuestionarios, estos pueden ayudar en el tamizaje de procesos o en su seguimiento evolutivo, disminuyendo el factor subjetivo del evaluador. Es por esto que en el proceso de transición, una de las recomendaciones, es contar con herramientas que permitan conocer el grado de preparación del paciente para el trasladado ${ }^{10,12}$. Existen variados instrumentos validados en distintas formas, enfocados a enfermedades crónicas genéricas o específicas, con diferentes números de preguntas y distintas escalas de respuesta. Algunos están diseñados para ser respondidos por los pacientes, otros por los padres y algunos por ambos ${ }^{13}$. Si bien es posible acceder a estos estudios, ningún instrumento ha sido desarrollado a nivel nacional. En español sólo existe la validación de la traducción argentina de un instrumento norteamericano (TRAQ-5), el que fue publicado durante la elaboración del presente estudio ${ }^{14}$. Es importante señalar que los instrumentos reportados en la literatura han sido creados en países desarrollados, lo que implica un contexto cultural diferente al local.

Este estudio tiene como objetivo crear un cuestionario que mida elementos importantes en la preparación de pacientes adolescentes con enfermedades crónicas para la transición a la atención de adultos, que sea acorde a nuestra realidad, que pueda ser aplicado de una forma fácil y comprensible para los jóvenes en un tiempo acotado, y que, finalmente pueda ser sometido a la metodología de validación de instrumentos.

Se aspira a contar con un primer instrumento que permita cumplir con una de las recomendaciones del proceso de transición ${ }^{10}$, y que al aplicarlo, este pueda contribuir a que los equipos tratantes tengan información general sobre el estado de sus pacientes previo a su traslado a servicios de adultos y así poder dirigir estrategias de mejoría en la práctica clínica o bien instaurar programas de transición que pueda ser medida con un instrumento estandarizado.

\section{Pacientes y Método}

Este estudio fue aprobado por el Comité de Ética Científico Pediátrico del Servicio de Salud Metropolitano Oriente. 
Tabla 1. Instrumentos validados para evaluar preparación en la transición

\begin{tabular}{|c|c|}
\hline Nombre del instrumento & Enfermedad objetivo \\
\hline TRAQ15 & Enfermedad crónica \\
\hline UNCTRXANSITION ${ }^{16}$ & Enfermedad crónica \\
\hline Self-management skills assessment guide ${ }^{17}$ & Enfermedad crónica \\
\hline TRANSITION-Q ${ }^{18}$ & Enfermedad crónica \\
\hline SCIS $^{19}$ & $\begin{array}{l}\text { Fibrosis quística con } \\
\text { insuficiencia pancreática }\end{array}$ \\
\hline $\mathrm{RTQ}^{20}$ & Trasplante renal \\
\hline $\mathrm{TRS}^{21}$ & Trasplante hepático \\
\hline
\end{tabular}

Tabla 2. Programas de transición disponibles en web

\begin{tabular}{|c|c|}
\hline Programas de transición & Página web \\
\hline Got Transition & http://www.gottransition.org/ \\
\hline $\begin{array}{l}\text { Jacksonville Health \& } \\
\text { Transition Services }\end{array}$ & http://www.hscj.ufl.edu.jaxhats/ \\
\hline $\begin{array}{l}\text { The Royal Children's } \\
\text { Hospital Melbourne }\end{array}$ & http://www.rch.org.au/transition/ \\
\hline $\begin{array}{l}\text { Transition to adult care: } \\
\text { Ready Steady Go }\end{array}$ & $\begin{array}{l}\text { http://www.uhs.nhs.uk/ } \\
\text { Ourservices/Childhealth/ } \\
\text { TransitiontoadultcareReadySteadyGo/ } \\
\text { Transitiontoadultcare.aspx }\end{array}$ \\
\hline $\begin{array}{l}\text { Children's Hospital at } \\
\text { Westmead Transitional } \\
\text { Care Policy }\end{array}$ & $\begin{array}{l}\text { https://www.schn.health.nsw.gov.au/ } \\
\text { hospitals/kids-and-teenagers/teenagers/ } \\
\text { adult-hospital }\end{array}$ \\
\hline On TRAC & http://www.ontracbc.ca \\
\hline
\end{tabular}

Se realizó una búsqueda en PubMed de instrumentos validados para evaluar a pacientes en su proceso de transición y se revisaron en detalle los artículos que se encontraban completos en dicha base de datos (tabla 1), a la vez se realizó una exploración en motor de búsqueda Google sobre programas de transición existentes en hospitales y se seleccionaron aquellos que tuviesen algún instrumento clínico para el mismo fin (tabla 2).

Tras un trabajo de lectura y discusión de los diferentes ítems, dimensiones y escalas de los cuestionarios encontrados, se diseñó un cuestionario nuevo. Este fue elaborado creando nuevas preguntas basadas en los distintos instrumentos, así como tomando y adaptando otras que se consideraron más pertinentes al contexto cultural.

Debido a que TRAQ- $5{ }^{15}$ y el cuestionario "Self Management checklist" del hospital pediátrico Westmead (tabla 2), fueron los instrumentos de los cuales se ob- tuvieron mayores insumos, el primero por sus fuertes pruebas de validación y el segundo por la concordancia cultural de varios de los ítems, se contactó a los autores vía correo electrónico obteniendo su permiso para el uso del material.

\section{Validez de contenido}

Se evaluó la validez de contenido por ítem de acuerdo a la metodología sugerida en Grant y Davis, basado en el trabajo de Hambleton y colaboradores, luego ampliado por Martuza y Waltz et al. ${ }^{22}$. Vía correo electrónico, 11 expertos aceptaron participar: 3 médicos especialistas en adultos (médicos internistas), 6 especialistas en adolescentes (pediatras adolescentólogos) y 2 expertos en medicina infantil (pediatras).

Los expertos, de forma independiente, revisaron cada uno de los ítems, evaluando su relevancia para el proceso de transición. Esto se realizó usando una escala de 4 puntos: $1=$ No relevante, $2=$ Poco relevante, 3 $=$ Bastante relevante y $4=$ Muy relevante ${ }^{22,23}$. Las respuestas por ítem fueron tabuladas en dos categorías, 'No relevantes-Poco relevantes' y 'Bastante relevantesMuy Relevantes'. Esta última categoría fue dividida por el total de respuestas, obteniéndose el índice de validez de contenido por ítem (IVC-I). Cada índice fue ajustado contra la probabilidad de que los expertos coincidan en su evaluación debido al azar, generando un nuevo índice "Kappa modificado" $\left(\mathrm{k}^{\star}\right)$ para cada ítem, los cuales fueron categorizados según los criterios establecidos por Polit, Beck, y Owen ${ }^{23}$.

A la vez se les solicitó que evaluaran cualitativamente el instrumento, por lo que se les pidió expresar sus opiniones sobre las distintas dimensiones e ítems. Se preguntó sobre edad y contexto de aplicación del instrumento, y comentarios generales.

Escala

Esta se realizó en base a las etapas del modelo transteórico del cambio, cuyo enfoque tiene como meta el cambio de conductas en salud, por lo que se ajustaría también para adquisición de nuevas habilidades en el cuidado y manejo de la enfermedad ${ }^{25}$. Esta escala fue la utilizada en el instrumento TRAQ-5, siendo validada dentro de su estudio ${ }^{15}$, pero se realizaron algunas modificaciones para que coincidieran gramaticalmente con los nuevos ítems y se solicitó a una experta en este modelo que avalara que mantuvieran reflejando dichas etapas.

\section{Reclutamiento de pacientes}

Muestra por conveniencia, sin estratificación. Se invitó participar a adolescentes que hubiesen acudido a una evaluación médica en el Hospital Luis Calvo Mackenna. Se les solicitó el asentimiento informado a ellos y el consentimiento informado a alguno de sus 
padres. Los criterios de inclusión fueron tener entre 12 a 19 años y padecer alguna patología crónica. El criterio de exclusión fue el tener algún déficit cognitivo evidente o cursar en el momento algún evento médico agudo que interfiriera en la actividad. Este proceso fue realizado por los autores y por un grupo de encuestadores.

El tamaño muestral necesario para validez de constructo y fiabilidad según Terwee es entre 100 y 250 cuestionarios, lo que se planificó alcanzar en un año ${ }^{26}$.

\section{Validación de comprensión y factibilidad}

Para evaluar factibilidad y comprensión se probó el instrumento en un grupo pequeño de pacientes, a modo de estudio piloto, con las mismas características señaladas anteriormente hasta alcanzar saturación de la información buscada.

Se le pidió a cada joven que llene por sí mismo el cuestionario tomándole el tiempo. Una vez realizado esto, se revisó ítem por ítem, solicitándole que explique lo que comprendió en cada pregunta. Se subrayaron las palabras que no entendían bien, se discutió junto con el joven el uso de sinónimos o una forma distinta de plantear la pregunta para una mejor comprensión de éstas. También se evaluó si tenían dificultades con la escala aplicada. Finalmente se les solicitó su percepción general sobre el instrumento.

\section{Validez de constructo y fiabilidad}

Los ítems que conformaban el cuestionario se agruparon según las diferentes dimensiones que teóricamente medían. Estas dimensiones fueron propuestas por los autores considerando lo aprendido de los instrumentos revisados y confirmados por la opinión de los expertos.

Se cargó el cuestionario en una Tablet utilizando la aplicación Teamscope (www.teamscope.co), que es una aplicación móvil de recolección de datos para investigación que permite la recopilación de datos sin conexión a internet. Posteriormente se les pidió a los pacientes que lo respondieran por sí mismos en este dispositivo dándoles a instrucciones iniciales.

Para confirmar la validez de constructo del instrumento, se realizó un análisis factorial exploratorio, utilizando una metodología de Principal Component Analysis $^{27}$, con el fin de detectar los ítems con baja comunalidad. Los ítems restantes fueron sometidos a la prueba de Kaiser-Meyer-Olkin y de Esfericidad de Bartlett ${ }^{28}$, para evaluar cuán adecuados son los datos resultantes de las pruebas anteriores para realizar el análisis factorial. En base a las pautas de Matsunaga ${ }^{29}$ se determinó la estructura de factores y finalmente se revisó la fiabilidad mediante consistencia interna de los componentes obtenidos de los análisis previamente descritos.

\section{Resultados}

Tras la revisión de literatura se logró la versión n ${ }^{\circ} 1$ del instrumento con 6 dominios: "actividades cotidianas" (3 ítems), "aspectos de mi enfermedad" (4 ítems), "manejo y uso de medicamentos" (4 ítems inicialmente), "aspectos prácticos de la atención de salud" (6 ítems), "participación en la consulta" (4 ítems) y "traslado" (3 ítems).

\section{Validez de contenido}

Dentro del IVC-I, se obtuvieron 20 ítems excelentes y 3 buenos. No cumplió el criterio de relevancia necesario el ítem 2 “Realizo tareas para ayudar en casa?".

Tras evaluar las sugerencias cualitativas de los jueces se decidió agregar una pregunta abierta para que los pacientes dejaran sus inquietudes. Además, se decidió separar en dos preguntas aquel ítem en que se preguntaba a la vez sobre si conocían los nombres de los medicamentos que usaban y para qué servían (versión $\mathrm{n}^{\circ} 2$, de 25 ítems más una pregunta abierta). Los expertos señalaron que el instrumento pudiese ser utilizado en pacientes de entre 12 y 18 años. Hicieron comentarios manifestando la utilidad de éste en la atención clínica para preparar la transición o al momento de la primera atención en servicios de adultos, pudiendo realizarse en el establecimiento, sala de espera o consulta, o vía correo electrónico previo a la consulta. Hubo dos sugerencias de aplicación paralela de un instrumento modificado para padres.

Tras la asesoría de un metodólogo externo se volvió a revisar el instrumento y se le realizaron modificaciones de redacción (versión $n^{\circ} 3$ ), manteniendo transitoriamente el ítem que no cumple criterios según expertos, para evaluar sus propiedades psicométricas (tabla 3). A esto se le agregó la escala de respuesta: 1. No, no lo necesito; 2. No, pero tal vez debiera; 3. Empezaré a hacerlo; 4. Sí, empecé a hacerlo hace poco; 5. Sí, hace más de 6 meses (Versión $n^{\circ} 4$ ).

\section{Validez de factibilidad y comprensión}

Entre junio y julio del 2016 se reclutaron 8 pacientes de 12 a 16 años para una prueba piloto. Todos aquellos adolescentes a los que se les pidió colaboración accedieron. Dentro de las patologías que portaban se encontraban: Vejiga neurogénica, trasplante renal, malformación ano-rectal, diabetes mellitus tipo 1 y depresión mayor. La versión $n^{\circ} 4$ del cuestionario pudo ser respondida por todos los participantes, tardando entre 6 y 10 minutos. Posteriormente se acogieron sus sugerencias de comprensión de palabras y de redacción obteniendo la versión $n^{\circ} 5$ del cuestionario. El formato de escala no tuvo sugerencias para ser modificada. 
Tabla 3. Media y desviación estándar (DS) por ítem y consistencia interna por dimensión

\begin{tabular}{|c|c|c|c|}
\hline Dimensión & Item & Media \pm DS & $\alpha$ de Cronbach \\
\hline \multirow{3}{*}{$\begin{array}{l}\text { Actividades } \\
\text { Cotidianas }\end{array}$} & 1. ¿Te responsabilizas de tus cosas personales? (Ordenar tu pieza, escolares, etc.) & $4,31 \pm 0,92$ & \multirow[t]{3}{*}{0,382} \\
\hline & $\begin{array}{l}\text { 2. ¿Realizo tareas para ayudar en casa? (poner la mesa, preparación de comidas, } \\
\text { limpieza general, etc.) * }\end{array}$ & $4,22 \pm 1,03$ & \\
\hline & 3. ¿Vas a comprar tú solo a tiendas, almacenes, farmacias u otros lugares? ${ }^{\circ}$ & $4,02 \pm 1,34$ & \\
\hline \multirow[t]{4}{*}{$\begin{array}{l}\text { Aspectos de mi } \\
\text { enfermedad }\end{array}$} & $\begin{array}{l}\text { 4. ¿Conoces tu historia médica? (Tus diagnósticos, si te han operado, hospitalizaciones, } \\
\text { alergias) }\end{array}$ & $4,62 \pm 0,86$ & \multirow[t]{4}{*}{0,537} \\
\hline & $\begin{array}{l}\text { 5. ¿Sabes bien de qué se trata tu enfermedad? (Síntomas, pronóstico y en qué te limita } \\
\text { esta) }\end{array}$ & $4,56 \pm 0,91$ & \\
\hline & 6. ¿Entiendes lo que causó tu enfermedad? & $3,95 \pm 1,32$ & \\
\hline & 7. ¿Realizas tú sólo el cuidado básico de tu enfermedad? & $3,60 \pm 1,42$ & \\
\hline \multirow{5}{*}{$\begin{array}{l}\text { Manejo } \\
\text { y uso de } \\
\text { medicamentos }\end{array}$} & 8. ¿Conoces el nombre de los medicamentos, tratamientos o procedimientos que usas? & $4,20 \pm 1,23$ & \multirow[t]{5}{*}{0,702} \\
\hline & 9. ¿Conoces para qué sirven los medicamentos, tratamientos o procedimientos que usas? & $4,35 \pm 1,12$ & \\
\hline & $\begin{array}{l}\text { 10. ¿Entiendes los efectos secundarios de los medicamentos que usas? (Otros efectos } \\
\text { que te pueda provocar, por ejemplo: dolor de estómago, problemas a los riñones, } \\
\text { subir la presión) }\end{array}$ & $3,19 \pm 1,50$ & \\
\hline & $\begin{array}{l}\text { 11. ¿Usas/tomas tus medicamentos, tratamientos o procedimientos por ti mismo de } \\
\text { forma correcta? }\end{array}$ & $4,01 \pm 1,34$ & \\
\hline & $\begin{array}{l}\text { 12. ¿Te preocupas de avisar cuando te quedan pocos medicamentos, dosis u otros } \\
\text { materiales? }\end{array}$ & $3,63 \pm 1,52$ & \\
\hline \multirow{6}{*}{$\begin{array}{l}\text { Aspectos } \\
\text { prácticos de } \\
\text { la atención de } \\
\text { salud }\end{array}$} & 13. ¿Sabes lo que debes hacer si te descompensas, te sientes mal o te enfermas? & $4,11 \pm 1,33$ & \multirow[t]{6}{*}{0,649} \\
\hline & 14. ¿Sabes a quién contactar en caso de emergencia? & $4,67 \pm 0,85$ & \\
\hline & 15. ¿Te encargas tú de pedir tus horas médicas? & $2,20 \pm 1,21$ & \\
\hline & 16. ¿Sabrías cómo llegar por ti mismo al hospital? & $4,10 \pm 1,33$ & \\
\hline & 17. ¿Tienes un sistema propio de registro y/o recordatorio de tus horas médicas? & $3,23 \pm 1,53$ & \\
\hline & 18. ¿Sabes cuál es tu plan de FONASA/ISAPRE u otro plan de salud y lo que este cubre? & $3,35 \pm 1,51$ & \\
\hline \multirow{4}{*}{$\begin{array}{l}\text { Participación } \\
\text { en la consulta }\end{array}$} & 19. ¿Eres tú el que responde las preguntas que hace el médico o profesional de salud? & $3,65 \pm 1,21$ & \multirow[t]{4}{*}{0,665} \\
\hline & $\begin{array}{l}\text { 20. ¿Serías capaz de entrar tú solo, o sea sin tus padres, a la consulta del doctor u otro } \\
\text { profesional de la salud? }\end{array}$ & $3,39 \pm 1,41$ & \\
\hline & $\begin{array}{l}\text { 21. ¿Te diriges al médico u otro profesional de la salud por tu propio interés, por } \\
\text { ejemplo haciendo preguntas o contándoles lo que te pasa o cómo te sientes? }\end{array}$ & $3,24 \pm 1,47$ & \\
\hline & 22. ¿Participas en la toma de decisiones con respecto a tu salud? & $3,67 \pm 1,39$ & \\
\hline \multirow[t]{3}{*}{ Traslado } & $\begin{array}{l}\text { 23. ¿Sabes a dónde y cuándo te trasladarán en el momento en que pases al sistema de } \\
\text { salud adulto? }\end{array}$ & $2,83 \pm 1,36$ & \multirow[t]{4}{*}{0,452} \\
\hline & 24. ¿Tienes un registro de tu historia médica y/o resumen para el traslado? & $3,23 \pm 1,48$ & \\
\hline & 25. ¿Te sientes listo para ser trasladado al sistema de salud adulto? & $2,49 \pm 1,21$ & \\
\hline $\begin{array}{l}\text { Pregunta } \\
\text { abierta }\end{array}$ & $\begin{array}{l}\text { 26. Si quieres, puedes dejarnos tus comentarios (preguntas, solicitudes y/o } \\
\text { preocupaciones) }\end{array}$ & & \\
\hline
\end{tabular}

*Relevancia insuficiente por jueces. ${ }^{\circ}$ Obtiene resultados psicométricos aceptables.

\section{Validez de constructo}

En el plazo de un año no se alcanzó la muestra esperada, por lo que se logró extender el estudio a 2 años, período en el cual se pudo reclutar 174 pacientes que respondieron la versión $n^{\circ} 5$ del cuestionario, principalmente en contexto de sala de espera. De estos, 168 (96\%) lo completaron, por lo que 6 cuestionarios fueron excluidos del análisis. La edad promedio fue 14,4 años $(\mathrm{SD}=1,66)$, con una mediana de 14 años (rango 12-19). 66\% correspondió a sexo femenino.

En total los pacientes identificaron 21 unidades clínicas en las cuales se atendían (Oncología, Broncopulmonar, Cirugía Plástica, Endocrinología, Nefrología, Inmunología y Gastroenterología entre otras). Las patologías reportadas con mayor frecuencia fueron asma, diabetes tipo 1, fisura labio palatina, trasplante 
hepático, patología oncológica tratada en etapa de seguimiento y enfermedad renal crónica.

La media y desviación estándar de los puntajes por cada ítem se reportan en la tabla 3, así como la consistencia interna de cada una de las dimensiones del instrumento.

Para establecer la estructura de factores del instrumento, se realizó un análisis factorial exploratorio ${ }^{30}$. Debido a que los datos violan el supuesto de distribución normal multivariante, se eligió el método de análisis de factor de eje principal para extraer los factores sobre los 25 ítems que componen el cuestionario, usando la muestra de 168 sujetos $^{31}$. La inspección de la matriz de correlaciones indicó que ciertos ítems no tuvieron ningún coeficiente de correlación por sobre \pm 0,3 , por lo que estos ítems fueron removidos y el Principal Component Analysis ejecutado nuevamente (27, 32), resultando en 7 iteraciones, eliminando los ítems $1,2,7,10,11,17,18,23,24,25$. Con los ítems restantes se realizaron 6 extracciones de factores consecutivas, descartando un ítem por iteración, usando como criterio una saturación inferior a $0,4^{27}$. Esto redujo el total a 9 ítems: $3,4,5,6,8,9,15,16,20$. La matriz de correlaciones indicó que todas las variables tuvieron al menos una correlación sobre 0,3 . La medida general de Kaiser-Meyer-Olkin (KMO) fue de 0,75 con medidas individuales de KMO 0,7 con excepción del ítem 3 que obtuvo un valor de 0,67. El resto de los índices clasifican como 'regular' a 'meritorio' ${ }^{28}$. La prueba de esfericidad de Bartlett fue estadísticamente significativa $(\mathrm{p}<0,0005)$, indicando que los datos son probablemente factorizables.
Para determinar la estructura de factores, se realizó un análisis paralelo siguiendo las pautas establecidas por Matsunaga ${ }^{29}$, revelando dos componentes que explicaron $31,2 \%$ y $17,6 \%$ de la varianza total, respectivamente. En conjunto, la solución de dos componentes explica un $48,8 \%$ de la varianza total. Para facilitar la interpretabilidad, se utilizó una rotación oblicua con método oblimin.

Analizando temáticamente los dos componentes de acuerdo a los ítems que mostraron mayor comunalidad, el Componente 1 captura ítems relacionados a "Aspectos de mi Enfermedad" y "Manejo y Uso de Medicamentos", mientras que el Componente 2 incluye "Aspectos prácticos de la atención de salud", "Participación en la consulta" y "Actividades Cotidianas". Las cargas factoriales o saturación junto a las comunalidades de la solución rotada están incluidas en la Tabla 4. Se revisó la consistencia interna de ambos componentes, dando para el Componente 1, el alfa de Cronbach $\alpha=0,68$ y para el Componente 2 alfa de Cronbach $\alpha=$ 0,66 . Estas indican una confiabilidad que limita con lo mínimamente aceptable (nivel débil).

\section{Pregunta abierta}

Sólo 18 de los 174 participantes utilizaron este segmento. El bajo número de respuestas y el que la mayoría de ellas fueran muy breves, no permite realizar un análisis profundo.

A continuación se describen los comentarios recibidos: 3 sujetos lo utilizaron para expresar haber tenido un mala experiencia en una atención en un hospital de adultos ( 2 por dificultad para conseguir hora y 1 por

Tabla 4. Matriz de estructura rotada para PAF (Principal Axis Factor) con una Rotación Oblicua con Método Oblimin de un cuestionario de dos componentes

\begin{tabular}{|c|c|c|c|}
\hline \multirow[t]{2}{*}{ Ítems } & \multicolumn{2}{|c|}{ Coeficientes de Componente Rotado } & \multirow[b]{2}{*}{ Comunalidades } \\
\hline & Componente 1 & Componente 2 & \\
\hline 5. ¿Sabes bien de qué se trata tu enfermedad? & $0,71 *$ & $-0,093$ & 0,476 \\
\hline $\begin{array}{l}\text { 9. ¿Conoces para qué sirven los medicamentos, tratamientos o procedimientos que } \\
\text { usas? }\end{array}$ & $0,580^{*}$ & 0,097 & 0,377 \\
\hline $\begin{array}{l}\text { 8. ¿Conoces el nombre de los medicamentos, tratamientos o procedimientos que } \\
\text { usas? }\end{array}$ & $0,543^{*}$ & 0,231 & 0,419 \\
\hline 4. ¿Conoces tu historia médica? & $0,518^{*}$ & 0,003 & 0,269 \\
\hline 6. ¿Entiendes lo que causó tu enfermedad? & $0,412^{*}$ & $-0,057$ & 0,160 \\
\hline 16. ¿Sabrías cómo llegar por ti mismo al hospital? & 0,204 & $0,679 *$ & 0,579 \\
\hline $\begin{array}{l}\text { 20. ¿Serías capaz de entrar tú solo, o sea sin tus padres, a la consulta del doctor } \\
\text { u otro profesional de la salud? }\end{array}$ & 0,018 & $0,609 *$ & 0,377 \\
\hline 15. ¿Te encargas tú de pedir tus horas médicas? & $-0,027$ & $0,585^{*}$ & 0,334 \\
\hline 3. ¿Vas a comprar tú solo a tiendas, almacenes, farmacias u otros lugares? & $-0,052$ & $0,407 *$ & 0,157 \\
\hline
\end{tabular}


trato deficiente); 3 manifestaron el deseo de prolongar la atención pediátrica más allá de los 15 años (hasta los 17, hasta los 18 y no trasladarse nunca); 3 propusieron un cambio en la modalidad de respuestas del cuestionario (a respuestas dicotómicas sí/no, incorporando a la escala de respuesta un "no me dejan", cambiar la escala a respuestas abiertas); 2 dan felicitaciones por el cuestionario; 2 refieren no conocer su diagnóstico final; 1 consulta sobre el cómo ayudaría el cuestionario al traslado a hospital de adultos; 1 manifiesta preocupación por el cambio de equipo médico debido al traslado; 1 manifiesta preocupación constante por su salud; 1 pregunta a qué edad es el traslado; 1 escribe un saludo informal.

\section{Instrumento final}

Se generó un cuestionario con 6 dimensiones y 24 ítems a los cuales se dio su numeración definitiva. Por la escasa utilización de la pregunta abierta y para hacer más liviano el instrumento, ésta no fue incluida. Se destacan las 9 preguntas que tuvieron pruebas psicométrica mínimamente aceptables resaltándolas en el instrumento final (figura 1).

\section{Discusión}

En Chile no hay una política universal de transición, existiendo sólo algunos esfuerzos locales para incorporar la preparación sistemática de este proceso. Durante el desarrollo de esta investigación se publicó el "Programa Nacional de Salud Integral de Adolescentes y Jóvenes: Nivel Especializado de Atención Abierta y Cerrada"33, en donde por primera vez se realizan recomendaciones ministeriales para abordar la transición, sumándose a lo ya realizado en otros países. En el programa se mencionan diversas actividades necesarias para preparar este proceso, encontrándose dentro de ellas el tener instrumentos para ir evaluando a los adolescentes con enfermedad crónica durante esta etapa, lo cual es el foco de esta investigación.

En este estudio se logra construir un instrumento con un conjunto de ítems adecuadamente validados en su contenido por expertos. En 9 de ellos se logra establecer que cumplen con los criterios mínimos de confiabilidad psicométricas para ser utilizados. No obstante, el haber mantenido exclusivamente dichos ítems hubiera implicado eliminar preguntas de alta relevancia clínica. Por ello se tomó la decisión de mantener los 24 ítems, pero destacando aquellos con mejor confiabilidad en los análisis de validez de constructo.

En base a los análisis realizados, recomendamos que investigaciones interesadas en continuar explorando las propiedades y características del instrumento, consideren que podrían haber existido eventuales dificultades en la comprensión lectora de la población evaluada y/o en la mantención de la atención frente a una gran cantidad de ítems. También podría haber jugado algún rol, el responder en el contexto de sala de espera con una alta interferencia ambiental, a diferencia de lo ocurrido en la prueba piloto.

A diferencia de encuestas tipo "check list" encontradas en programas hospitalarios, la escala graduada obtenida, permite obtener matices más allá de "logrado" o "no logrado", lo que posibilita hacer un seguimiento más detallado del paciente al administrar la prueba en forma seriada. Más aún considerando que la escala basada en el modelo transteórico del cambio permite evaluar y trabajar la disposición frente al cambio en conductas de salud ${ }^{25}$. No obstante, si bien esto se puede considerar una ventaja, también es posible que la complejidad de la escala haya contribuido al mal desempeño psicométrico de las preguntas. En particular, las diferencias con los resultados obtenidos en el estudio de TRAQ- $5^{15}$ y también de su traducción argentina ${ }^{14}$, pudieran explicarse debido a que ambos instrumentos fueron validados en un grupo de pacientes de edades mayores, 16 a 26 años el primero, y mayores de 14 años el segundo ${ }^{15}$, lo que tendría una utilidad limitada para el contexto de salud chileno, donde el traslado sucede a los 15 años.

La validación de un instrumento es un proceso continuo y dinámico que va adquiriendo más peso cuantas más propiedades psicométricas se hayan medido en distintas culturas, con distintas poblaciones y sujetos. A pesar de las limitaciones planteadas, creemos que este trabajo contribuye con los primeros pasos en la elaboración de un instrumento acorde a nuestra realidad local, obteniéndose un conjunto de preguntas de relevancia clínica, validadas por expertos, que ameritan ser administradas a los pacientes para preparar su transición. Aunque en términos generales el instrumento tiene problemas importantes de confiabilidad psicométrica, este estudio presenta información detallada respecto a las propiedades del instrumento, complementando la investigación realizada a la fecha ${ }^{13}$. También vemos como ventaja el que esté dirigida a pacientes con enfermedad crónica en forma genérica, ya que permite abarcar un amplio abanico de patologías. Además su formato de auto-reporte, simplifica su implementación. Esta investigación permite establecer los primeros pasos hacia encontrar variables que puedan ser clínicamente relevantes para la predicción de éxito de la transición.

El trabajo clínico en transición debe ir centrado en las necesidades del paciente ${ }^{9-12}$, y este instrumento es una forma de visualizar elementos importantes de los pacientes durante ésta. Si bien este instrumento no logró la validacion, contiene elementos que de acuerdo a nuestra experiencia clínica, consideramos de utilidad 


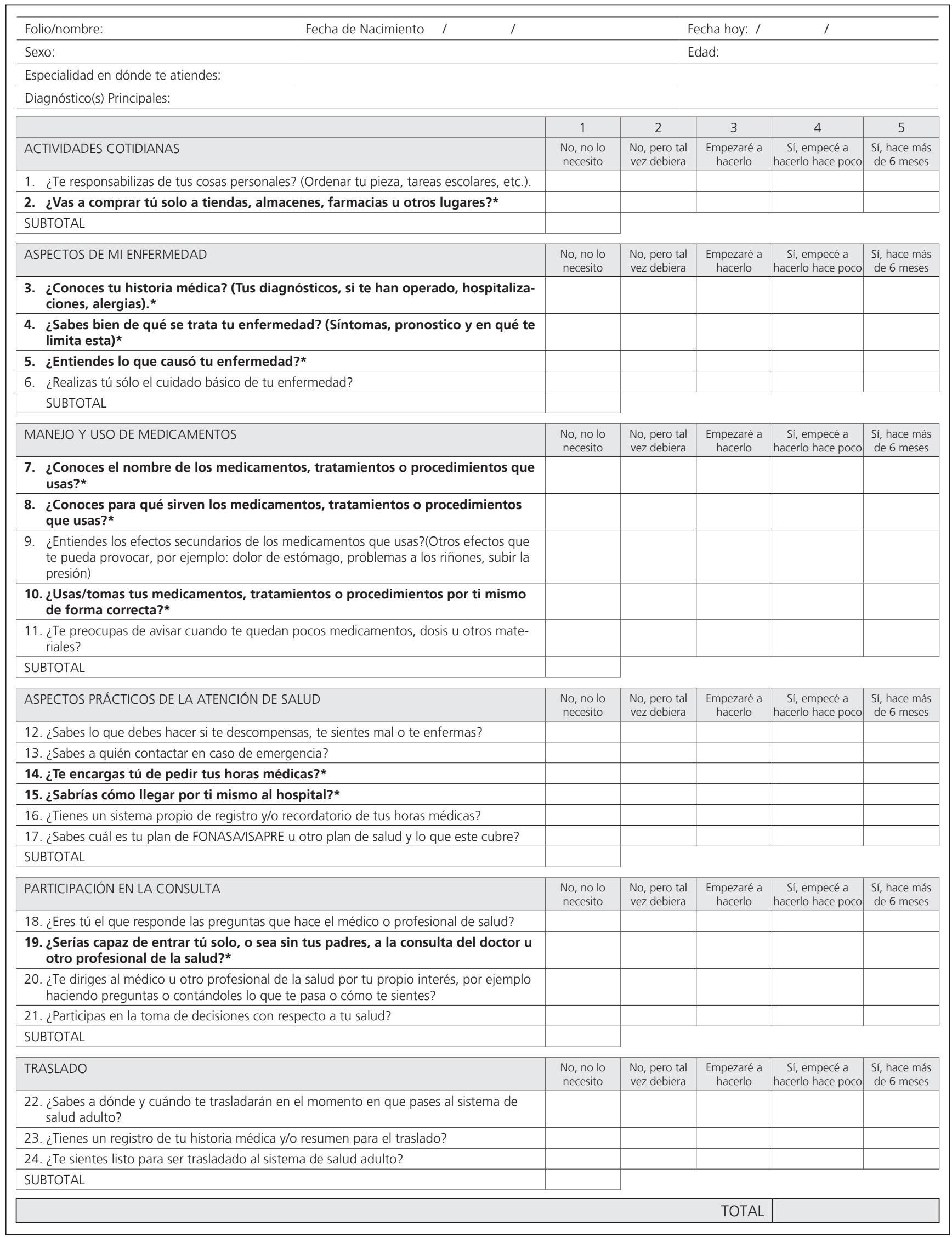

Figura 1. Cuestionario de evaluación del estado para la transición. *ítems que cumplen con los criterios mínimos de confiabilidad psicométrica. 
para aplicarlo en unidades clínicas y/o trabajarlo directamente con los adolescentes en las atenciones de salud, conociendo el proceso del paciente que tenemos en frente y sus áreas a reforzar. De esta forma pudiera ser utilizado como un instrumento de evaluaciónintervención por los equipos clínicos, considerando las limitaciones que la falta de validación representa..

En conclusión, el análisis permitió construir un cuestionario de auto-reporte para medir el estado de preparación de los adolescentes para la transición, basado en cuestionarios internacionales, con un contenido validado por jueces locales, y factible de aplicar en un tiempo acotado. Se reportan las propiedades psicométricas del instrumento comprobando la validez de constructo y confiabilidad para 9 de los 24 ítems, lo cual representa una insuficiente validación que debe ser considerada al momento de su aplicación en la practica clínica.

\section{Responsabilidades Éticas}

Protección de personas y animales: Los autores declaran que los procedimientos seguidos se conformaron a las normas éticas del comité de experimentación humana responsable y de acuerdo con la Asociación Médica Mundial y la Declaración de Helsinki.

Confidencialidad de los datos: Los autores declaran que han seguido los protocolos de su centro de trabajo sobre la publicación de datos de pacientes.
Derecho a la privacidad y consentimiento informado: Los autores han obtenido el consentimiento informado de los pacientes y/o sujetos referidos en el artículo. Este documento obra en poder del autor de correspondencia.

\section{Agradecimientos}

Médicos Expertos: Loreto Podestá; Tamara Zubarew; Verónica Gaete; María Eugenia Henríquez; Francisca Salas; Gonzalo Menchaca; Fernando Barahona; Macarena Armstrong; Poletnzi Uriarte; Eduardo Wolff; Francisco Cano.

Experta en modelo del cambio: Carolina López.

Experta en metodología: Lorena Reinike.

Reconocimiento de contribuciones que no alcanzan autoría: Metodólogo Gonzalo López.

\section{Financiamiento}

Concurso anual de investigación Hospital Luis Calvo Mackenna, Departamento de Pediatría y Cirugía infantil Oriente año 2015.

\section{Conflicto de intereses}

Los autores declaran no tener conflicto de intereses.

\section{Referencias}

1. Vargas N, Quezada A. Epidemiología, nueva morbilidad pediátrica y rol del pediatra. Rev Chil Pediatr. 2007;78(1):103-10.

2. Huang JS, Gottschalk M, Pian M, Dillon L, Barajas D, Bartholomew LK. Transition to adult care: systematic assessment of adolescents with chronic illnesses and their medical teams. J Pediatr. 2011;159(6):994-8.

3. Inostroza C, Correa ML, Besoaín C, et al. El proceso de transición de servicios pediátricos a adultos: visión de adolescentes hospitalizados portadores de enfermedades crónicas. Rev Chil Pediatr. 2016;87(2):110-5.

4. Pacaud D, Yale JF, Stephure D, Truselle $\mathrm{R}$, Davies HD. Problems in Transition From Pediatric Care to Adult Care for Individuals With Diabetes. Canadian journal of Diabetes. 2005;29(1):13-8.

5. Lam PY, Fitzgerald BB, Sawyer SM. Young adults in children's hospitals: why are they there?. Med J Aust.
2005;182(8):381-4.

6. Geerts E, van de Wiel H, Tamminga R. A pilot study on the effects of the transition of paediatric to adult health care in patients with haemophilia and in their parents: patient and parent worries, parental illness-related distress and health-related Quality of Life. Haemophilia. 2008;14(5):1007-13.

7. Wolf-Branigin M, Schuyler V, White P. Improving quality of life and career readiness of adolescents with disabilities: experiences from the Adolescent Employment Readiness Center. Res Soc Work Pract. 2007;17(3):324-33.

8. Gaete V, Henríquez ME, Robledo P, et al. Fundamentos para la extensión de la edad pediátrica hasta el término de la adolescencia a nivel de toda la red asistencial de salud. Recomendación del Comité de Adolescencia de la Sociedad Chilena de Pediatría. Rev Chil Pediatr. 2011;82(5):447-53.

9. American Academy of Pediatrics, American Academy of Family Physicians, American College of Physicians-American
Society of Internal Medicine. A Consensus statement on health care transitions for young adults with special health care needs. Pediatrics. 2002;110:1304-6.

10. American Academy of Pediatrics, American Academy of Family Physicians, American College of Physicians, Transitions Clinical Report Authoring Group, Cooley W C, Sagerman P J. Supporting the health care transition from adolescence to adulthood in the medical home. Pediatrics. 2011;128(1):182-200.

11. Lizama M, Ávalos M, Vargas N, Varela M, Navarrete C, Galanti M, Orellana J. Transición al cuidado de la vida adulta, de niños y adolescentes con necesidades especiales de atención en salud: recomendaciones del comité NANEAS de la Sociedad Chilena de Pediatría. Rev Chil Pediatr. 2011;82(3):238-44.

12. Zubarew T, Correa L, Bedregal P, et al. Transición de adolescentes portadores de enfermedades crónicas desde servicios pediátricos a servicios de adultos. Recomendaciones de la Rama de Adolescencia de la Sociedad Chilena 
de Pediatría. Rev Chil Pediatr. 2017; 88(4):553-60. DOI: 10.4067/S037041062017000400018.

13. Zhang L, Ho J, Kennedy S. A systematic review of the psychometric properties of transition readiness assessment tools in adolescents with chronic disease. BMC Pediatrics. 2014; 14: 4.

14. González F, Roizen M, Rodríguez M, et al. Validación español-argentina del cuestionario de transición a la atención médica del adulto en adolescentes con enfermedades crónicas. Arch Argent Pediatr 2017;115(1):18-27/18

15. Wood DL, Sawicki GS, Miller D, et al. The Transition Readiness Assessment Questionnaire (TRAQ): Its Factor Structure, Reliability, and Validity. Academic Pediatrics. 2014;14:415-22.

16. Ferris M, Harward D, Bickford K, et al. A clinical tool to measure the components of health-care transition from pediatric care to adult care: the UNC TR(x) ANSITION scale. Ren Fail. 2012;34:74453.

17. Williams T, Sherman E, Dunseith C, et al. Measurement of medical selfmanagement and transition readiness among Canadian adolescents with special health care needs. Int J Child Adolesc Health. 2010;3:527-35.

18. Klassen AF, Grant C, Barr R, et al. Development and validation of a generic scale for use in transition programmes to measure self-management skills in adolescents with chronic health conditions: the TRANSITION-Q. Child
Care Health Dev. 2015;41(4):547-58.

19. Patton SR, Graham JL, Varlotta L, Holsclaw D. Measuring self-care independence in children with cystic fibrosis: the self-care independence scale (SCIS). Pediatr Pulmonol. 2003; 36:12330.

20. Gilleland J, Amaral S, Mee L, Blount R. Getting ready to leave: transition readiness in adolescent kidney transplant recipients. J Pediatr Psychol. 2012;37:8596.

21. Fredericks EM, Dore-Stites D, Well A, et al. Assessment of transition readiness skills and adherence in pediatric liver transplant recipients. Pediatric Transplant. 2010;14:944-953.

22. Grant JS, Davis LL. Selection and use of content experts for instrument development. Research in Nursing \& Health. 1997;20(3):269-74.

23. Davis LL. Instrument review: Getting the most from a panel of experts. Applied Nursing Research. 1992;5(4):194-7.

24. Polit DF, Beck CT, Owen SV. Is the CVI an Acceptable Indicator of Content Validity? Appraisal and Recommendations. Research in Nursing \& Health. 2007;30:459-67.

25. Prochaska JO. Decision making in the transtheoretical model of behavior change. Medical Decision Making. 2008;28(6):845-9.

26. Terwee CB, Bot SD, de Boer MR,et al. Quality criteria were proposed for measurement properties of health status questionnaires. J Clin Epidemiol.
2007;60(1):34-42.

27. Gie Yong A, Pearce S. A Beginner's Guide to Factor Analysis: Focusing on Exploratory Factor Analysis. Tutorials in Quantitative Methods for Psychology. 2013;9(2):79-94.

28. Kaiser HF. An index of factorial simplicity. Psychometrika. 1974;39:32-6.

29. Matsunaga M. How to factor-analyze your data right: Do's, don'ts, and how-to's. International Journal of Psychological Research. 2010;3(1):97-110.

30. Flora DB, Flake JK. The purpose and practice of exploratory and confirmatory factor analysis in psychological research: Decisions for scale development and validation. Canadian Journal of Behavioural Science 2017;49(2):78-88.

31. Fabrigar LR, Wegener DT, Maccallum RC, Strahan EJ. Evaluating the Use of Exploratory Factor Analysis in Psychological Research. Psychological Methods. 1999;4(3):272-99.

32. Lloret-Segura S, Ferreres-Traver A, Hernández-Baeza A, Tomás-Marco I. (2014). El Análisis Factorial Exploratorio de los Îtems: una guía práctica, revisada y actualizada. Anales de Psicología. 2014;30(3):1151-69.

33. MINSAL 2018. Programa nacional de salud integral de adolescentes y jóvenes: nivel especializado de atención abierta y cerrada. Disponible en: https://diprece.minsal.cl/wpcontent/uploads/2018/12/2018.12.13 PROGRAMA-ADOLESCENTES_web. pdf. 